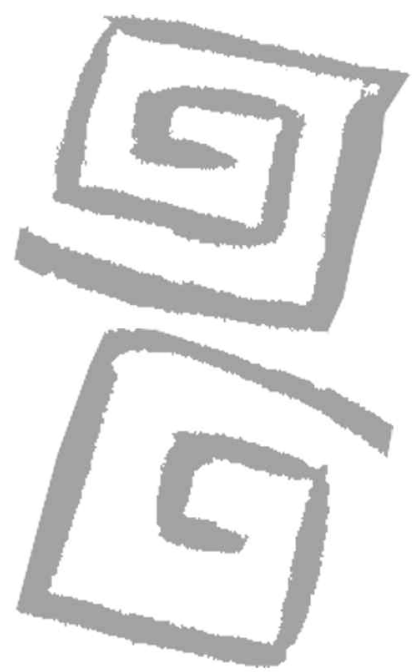

\title{
Evaluación de una propuesta pedagógica de enseñanza de la Medicina
}

\author{
Evaluation of a pedagogical proposal \\ in teaching Medicine
}

Silberman, Martín Salvador ${ }^{1}$; Silberman, Pedro ${ }^{2}$; Pozzio, María ${ }^{3}$

\footnotetext{
${ }^{1}$ Médico. Doctorando en Ciencias de la Salud, Gestión y Políticas Públicas. Profesor Departamento de Salud Pública, Facultad de Medicina, Universidad Nacional Autónoma de México (UNAM).

silbermanmartin@gmail.com

${ }^{2}$ Médico. Magíster en Salud Pública. Profesor Adjunto de Salud Individual y Colectiva, Departamento de Ciencias de la Salud, Universidad Nacional del Sur, Argentina. psilberman@uns.edu.ar

${ }^{3}$ Licenciada en Sociología. Magíster en Antropología Social. Auxiliar docente, Cátedra de Antropología Cultural y Social, Facultad de Psicología, Universidad Nacional de La Plata, Argentina. mariapozzio@gmail.com
}

RESUMEN En el presente artículo nos proponemos discutir el papel que cumplen las escuelas de medicina en la formación del perfil de los profesionales. Para ello, se analiza el impacto de dos experiencias de prácticas en terreno realizadas en 2006 y 2009, por los alumnos de la cátedra de Epidemiología de la Facultad de Ciencias Médicas de la Universidad Nacional de La Plata (Provincia de Buenos Aires, Argentina). Mediante cuestionarios semiestructurados individuales aplicados a los alumnos y estrategias de grupos focales se buscó obtener información de las ideas y representaciones de los estudiantes, antes y después de las experiencias pedagógicas. Entre los principales resultados se destaca la posibilidad de que los alumnos se replanteen los modelos explicativos del proceso salud-enfermedad-atención y el peso real de los problemas sociales sobre los fenómenos de salud y enfermedad.

PALABRAS CLAVE Escuelas de Medicina; Práctica Profesional; Condiciones Sociales; Argentina.

ABSTRACT In this article we discuss the role that medical schools play in the creation of a particular profile of health professionals. To this end, we analyze the impact of two field experiences carried out in 2006 and 2009 with students in the Epidemiology course of the Faculty of Medical Sciences in the Universidad Nacional de La Plata (in the province of Buenos Aires, Argentina). Using individual semistructured questionnaires applied to students as well as focus group strategies, the study sought to obtain information about the ideas and representations of the students before and after these educational experiences. The ability of students to reconsider the explicative models of the health-disease-care process and the weight of social problems in the phenomena of sickness and health is highlighted as one of the study's primary results.

KEY WORDS Medical Schools; Professional Practice; Social Conditions; Argentina. 


\section{INTRODUCCIÓN}

Las escuelas de medicina son las proveedoras del recurso humano prioritario de los sistemas de salud: los profesionales. Por lo tanto, no están fuera de las discusiones en torno al papel que juegan los sistemas en la determinación de la salud de los individuos, grupos y comunidades. Por ello es que tienen un gran desafío por resolver: ¿qué tipo de profesional formar, para qué tipo de práctica y en qué sistema ideal de inserción?

Los sistemas de salud son centro de debates permanentes en torno a su organización, efectividad y financiación. Dichos debates se actualizan en la medida en que los sistemas de salud no consiguen responder adecuadamente a los nuevos y complejos problemas que afectan a las sociedades. Las características de estos problemas y la complejidad de las estructuras sociales y políticas de los diferentes países y regiones han configurado un mosaico irregular de respuestas sanitarias en función de diferentes concepciones de lo que es la salud y la organización estatal de su cuidado.

Por otro lado, las universidades en las que funcionan las escuelas de medicina tienen estructuras características (1) que definen perfiles propios, muchas veces alejados de los problemas de salud y de las organizaciones encargadas de su resolución. Esto es visto, por un lado, como una ventaja en la formación de profesionales en lo relativo a la autonomía y libertad de pensamiento y, por otro, como un aspecto negativo, criticándole a las universidades su escaso compromiso en el desarrollo social y político de las sociedades que les dan sustento (2).

En el presente artículo nos proponemos discutir el papel que cumplen las escuelas de medicina en la formación del perfil de profesionales, es decir, cómo estas escuelas van construyendo la idea de lo que un profesional debe ser. Para llevar a cabo ese objetivo, hemos considerado pertinente utilizar algunos conceptos de Pierre Bourdieu a fin de generar un análisis que interpele al campo de la salud pública. Así, consideraremos a la salud pública como un campo en el que coexisten diferentes modelos médicos en pugna por la hegemonía (3). En la idea de campo (a) podemos observar la existencia de capitales en juego: el capital simbólico y el capital cultural (b) -que definen el saber y/o paradigma sobre lo que debe ser la salud y lo que debe ser un médico, saberes consagrados en títulos, credenciales y prestigio. La posesión y administración de estos capitales posibilita a su vez el control y acceso al capital económico, recursos, etc. A su vez, estos capitales son disputados por distintos actores que, con distintos intereses, pelean por esos recursos y la definición y resolución de los problemas propios del campo. Entonces, cada uno de los actores (políticos, académicos, profesionales, corporaciones) lucha por la consolidación, o por la apropiación y el predominio de uno o más de los capitales en pugna. A través de esta categoría de Bourdieu, podemos ver las reglas propias que rigen en el campo de la salud y la gran autonomía relativa que el mismo posee. De allí que para modificar posiciones, los actores deben aprehender y aceptar las reglas del juego y, sobre esa base, acumular capitales con las lógicas propias del campo (4).

Como plantea Bourdieu (5), es importante definir las posiciones que ocupan los agentes en cada campo (c). En el campo que nos ocupa, estas posiciones van desde jerarquías instituidas como dominantes -por ejemplo, decanos, directores de hospital, sociedades científicas, etc.- a otras que aparecen como subordinadas respecto de aquellas -trabajadores del primer nivel de atención, federaciones médicas, centros de estudiantes, etc.-, en función de los diferentes tipos y cantidad de capitales (económico, social, cultural, simbólico) puestos en juego en cada situación concreta.

En este sentido, plantear la noción de campo de salud permite ubicar al Modelo Médico Hegemónico, tal como es caracterizado por Eduardo Menéndez (6), como el modelo de quienes tienen la posición dominante del campo y pretenden, entre otras cosas, reducir las interpretaciones de la salud al radio de acción del individuo y a sus elecciones. En el otro polo del campo, entre quienes disputan la hegemonía del mismo, en una posición subalterna y hasta marginal, los actores esgrimen representaciones de salud que se encuentran fuertemente posicionadas en lo colectivo (7) (d).

Esta noción colectiva de la salud otorga un lugar central a los determinantes sociales de la 
salud $(8,9)$, a los que sitúa en el campo de la lucha política -en términos de Bourdieu, en el campo de poder- que es donde se configuran y distribuyen dichos determinantes. Por lo tanto, para las posiciones subalternas dentro del campo de la salud, esos determinantes van mucho más allá de los aspectos biofisiológicos del individuo -como lo plantearían las posiciones hegemónicas-, por cuanto limitarse a estos aspectos es una forma reduccionista de abordar la complejidad de los problemas de salud y enfermedad (10).

Es importante señalar que los sistemas de salud reproducen los modelos hegemónicos mediante su accionar cotidiano, moldeando las ideas de lo que un profesional de la salud debe ser, tanto al interior de las organizaciones formadoras, como en la propia comunidad. Generar un modelo como hegemónico justamente significa eso: que todos los actores, consciente o inconscientemente, hacen suyo ese modelo y así lo replican (11).

Por ello es tan difícil modificar esa situación, es decir, romper con la reproducción e introducir modificaciones que trabajen en la reorientación de los sistemas de salud y la formación de sus profesionales hacia un modelo más social. Estas modificaciones deben partir también de las escuelas de medicina, desafiando el perfil de formación profesional, lo que contribuiría a romper con la espiral reproductivista (e) que se da actualmente entre las escuelas de medicina y los sistemas de salud.

El presente trabajo analiza los impactos de una propuesta de enseñanza en la carrera de Medicina en los diferentes niveles de la formación médica y su capacidad de reorientarla hacia el componente social, histórico y cultural que determina los procesos salud-enfermedad en personas, grupos y comunidades; propuesta que debe ser entendida también en el marco de la disputa dentro del campo de la salud, como una estrategia de acumular capital social y simbólico en aras de fortalecer las posiciones de los actores que intentan revertir la hegemonía del campo.

La propuesta utiliza la herramienta de enseñanza vinculada a la práctica en terreno como un modo de adquisición de aprendizajes significativos en escenarios reales, donde se desarrollan y cobran sentido las ideas de los "determinantes sociales" en la producción de los procesos salud-enfermedad-atención.

\section{Modelos médicos}

Es posible señalar que el modelo de formación liberal, biologicista y acrítico de la práctica de la profesión médica se observa con regularidad en las diferentes escuelas de medicina de Argentina y se enmarca dentro de lo que se ha denominado como Modelo Médico Hegemónico $(\mathrm{MMH})(6,12)$. El MMH se genera y se reproduce en el seno de la formación médica, a través de la práctica docente de médicos y no médicos, y tiene su continuidad en la práctica profesional, construyendo así una hegemonía que intenta excluir y marginar otros modelos de atención.

Al sostenerse como dominante a lo largo del tiempo, ha generando en el campo de la salud lo que Bourdieu denomina habitus:

\footnotetext{
...sistemas de disposiciones duraderas y transferibles, estructuras estructuradas predispuestas para funcionar como estructuras estructurantes, es decir, como principios generadores y organizadores de prácticas y representaciones que pueden estar objetivamente adaptadas a su fin sin suponer la búsqueda consciente de fines y el dominio expreso de las operaciones necesarias para alcanzarlos, objetivamente "reguladas" y "regulares" sin ser el producto de la obediencia a reglas, y, a la vez que todo esto, colectivamente orquestadas sin ser producto de la acción organizadora de un director de orquesta. (13 p.92)
}

Este habitus es reproducido permanentemente por los actores universitarios a través de las prácticas docentes. Allí se ubica uno de los escenarios de disputa del campo de la salud, en el que se estructuran las ideas sobre lo que la práctica profesional ha de ser, ideas que son incorporadas por los estudiantes. Es así como alumnos y docentes van naturalizando esta forma de entender el ejercicio de la medicina que es parte constitutiva de su experiencia cotidiana, reproduciendo con sus representaciones, actitudes y prácticas, la hegemonía del modelo asistencial (11).

De esta manera, la formación de profesionales de la salud dentro de las escuelas de medicina se configura como una arena específica del campo de la salud. Es en el ámbito de la formación donde cada actor realiza su juego en función de incrementar y/o adquirir los capitales que 
pueden ser tanto simbólicos como sociales o económicos (horas docentes, cargos jerárquicos, financiamiento, mayor cantidad de materias o capacidad de gestión), cuya adquisición y/o acumulación les sirven para posicionarse en el campo más amplio de la salud. Es en esta arena específica -la formación de médicos- donde debemos generar mecanismos para dar un debate en torno a las posiciones de los modelos y en ese sentido se presenta la propuesta de enseñanza que analizamos en este artículo.

\section{Redefinición del profesional médico}

La definición del perfil de egresado de las escuelas de medicina se encuentra en permanente debate $(14,15)$. Por un lado, una minoría de actores universitarios y profesionales actúan por fuera de los claustros, traccionando hacia el objetivo de formar egresados más sensibles a la realidad $-\mathrm{O}$ a las necesidades que la sociedad requiere- o proponiendo capacitación permanente en salud (16). Por otro lado, los herederos de la tradición hegemónica (f) (directivos de las corporaciones profesionales y universitarias) rechazando estos intentos y conservando las relaciones de poder tal como están. Y en medio de estos dos sectores en pugna por la hegemonía del campo -los que la tienen y luchan por no perderla y los que la disputan, ofreciendo un modelo contrahegemónico- están los otros actores del campo que si bien no podemos considerar como parte de los herederos de la tradición, sí contribuyen a su reproducción.

Para comprender esto, es central el concepto de habitus (13): la mayoría de los miembros del campo quizá no se ven beneficiados -ni material ni simbólicamente- con la hegemonía actual, es decir, con un modelo de profesional especializado, liberal y acrítico. Sin embargo, al haber sido socializados en el campo, al pertenecer al mismo, son moldeados por el habitus que naturaliza ese modelo de profesional como el único posible (17). Como plantea Bourdieu (13), los habitus son "estructuras estructurantes" porque nos permiten percibir, aprehender el mundo $y$, por ende, actuar (18); y estructuradas, ya que vienen dadas y, por lo tanto, parecen obvias y naturales. Esta última característica es la que permite pensar en la transformación de esos habitus, sin lo cual no sería posible la lucha y la transformación de la hegemonía en el campo.

La ausencia de una mirada más humana, integral y holística de la medicina por parte de los egresados tiene en parte su origen en la mirada recortada y restringida de las escuelas formadoras y en las escasas oportunidades brindadas por dichas escuelas para permitir un contacto más amplio con la realidad social (19). Este "contacto" con la realidad social -donde se produce el proceso de salud-enfermedad-atención (PSEA) - es un punto clave desde el cual generar un diálogo entre la realidad y la teoría, diálogo que permita sensibilizar al estudiante a través de su experiencia. Consideramos esa experiencia no solo como lo vivido y percibido de forma primaria y sensible, sino como la mediación de eso vivido, que ya contiene de por sí una reflexión que articula el relato que se hace de esa misma experiencia y, por lo tanto, la complejiza (20). Esta experiencia de contacto con la realidad pone al docente y al alumno en un punto de reflexión y articulación de la teoría y la práctica, conceptos fundamentales de la propuesta analizada.

En el modelo vigente, la necesidad de la práctica en la carrera de Medicina no está puesta en duda, pero se la reduce a la adquisición de conocimientos y habilidades técnicas necesarias para realizar prácticas específicas vinculadas exclusivamente a tratamientos o procedimientos sobre los cuerpos de los pacientes. Se enseña a tomar la presión o suturar una herida, pero se desconoce la dimensión "actitudinal" como, por ejemplo, las reacciones e interacciones con el paciente.

Para incorporar lo actitudinal a la enseñanza se debe vincular teoría y práctica con el fin de desarrollar un conocimiento practicum:

\footnotetext{
...situación pensada y dispuesta para aprender una práctica en la que el estudiante aprende a evaluarla, debe diseñar y realizar su propia percepción de ella, reflexiona sistemáticamente sobre lo realizado y sus fundamentos, analiza casos o zonas indeterminadas de la misma. (21 p.253)
}

Este punto resulta clarificador del papel que deben desempeñar en el currículo las prácticas del trabajo en terreno, como un espacio de 
contacto y de reflexión de la práctica y no limitado al aprendizaje de un acto mecánico. De acuerdo a esto, se deben recuperar las experiencias ligadas a la práctica, para encuadrar los resultados obtenidos y poder producir un conocimiento sistemático sobre lo que implica enseñar y aprender una práctica profesional. Por ello es "necesario trabajar en una lógica de problemas y no una lógica de soluciones", haciendo que los problemas hallados en las prácticas "provoquen a los textos" (3 p.279).

En este marco, los objetivos del presente trabajo son: por un lado, determinar cómo se hacen efectivas las diferencias de capitales entre un modelo médico y en el ámbito de la formación de profesionales, por ejemplo, a través de la distribución porcentual de materias "duras" en el currículo, en cantidad de horas obligatorias de cursada, o en la estructura docente. Por otro lado, conocer si una propuesta pedagógica con una fuerte impronta en la determinación social de los fenómenos de salud-enfermedad logra impactar a nivel de las ideas preconcebidas y, de esa forma, en el habitus de los alumnos, para generar conciencia de la necesidad de reorientar los recursos y los capitales social y simbólico hacia otro modelo profesional.

\section{ASPECTOS METODOLÓGICOS}

Para tener elementos de análisis de la estructura de la carrera y de su orientación, se ha realizado, en primer lugar, un análisis cuantitativo de la estructura y distribución de recursos al interior de las cátedras. Por otro lado, se recabó información de las ideas que los propios alumnos esgrimen acerca de la organización del currículo y del modelo profesional antes de la implementación de la propuesta pedagógica basada en prácticas en terreno, realizadas desde la cátedra de primer año de Epidemiología. Luego de finalizadas las mismas, se procedió a evaluar el impacto que sobre esas ideas pueden tener dichas prácticas.

\section{Análisis de la estructura de la carrera}

En primer lugar, se realizó un análisis cuantitativo del currículo de formación de los médicos, mediante un análisis estructural de las materias incluidas en el Plan de estudios 2004 de la carrera de Medicina de la Facultad de Ciencias Médicas de la Universidad Nacional de La Plata (22), a las que se le asignaron dos categorías principales - "duras" y "sociales" (g) - según su objeto de estudio. Por fuera de esta clasificación colocamos a los talleres de integración básico-clínicos, que tienen por finalidad articular conocimientos del ciclo básico y clínico mediante la presentación de casos clínicos que incorporen conceptos de ambas fases del currículo.

Las materias consideradas "duras" son aquellas que tienen su base en los procesos biológicos que asientan en el cuerpo sin tener elementos teóricos relacionados con otras variables que expliquen los fenómenos de salud-enfermedad. En los discursos de estas materias, la enfermedad "comienza" y "ataca" por factores externos a la voluntad, como si fueran fenómenos naturales sin relación con determinantes sociales o culturales. Este discurso se da en materias troncales como Fisiología, Bioquímica, Anatomía, Patología, Clínica, y todas las materias del ciclo clínico. En contraposición, se categorizaron como "sociales" a aquellas materias que en su programa introducen contenidos relacionados con las ciencias sociales para explicar y complejizar el fenómeno de saludenfermedad. Para ello se analizaron los programas de contenidos de dichas materias, para poder categorizarlas de acuerdo a los objetivos del trabajo.

Una vez categorizadas las materias incluidas en el plan de estudios, se contabilizó la cantidad de materias "duras" y "sociales", la cantidad de horas asignadas a cada una de ellas y cuáles son consideradas obligatorias para cumplir con los contenidos curriculares. Sumado a ello, se buscó identificar las diferencias entre estas materias en función de la cantidad y jerarquía de sus planteles docentes. De esta manera, se intentó identificar niveles de importancia implícitos de las diferentes materias mediante el otorgamiento de la mayor estructura.

\section{Análisis cualitativo: ideas de los alumnos}

Es substancial sumar al diagnóstico las ideas y representaciones que tienen los alumnos acerca de la profesión, de su importancia en la 
sociedad y de los conocimientos que debe brindarles la facultad. Esto suma información esencial acerca de cómo forma su perfil profesional, rescatando elementos que el mismo alumno considera fundamentales para definirse como buen médico, para desde allí inferir cómo la organización del currículo de la facultad genera no solo conocimientos y herramientas sino también valoraciones y niveles jerárquicos de importancia entre las materias, consideraciones que terminan impactando en el habitus de los mismos.

Para ello hemos recabado información correspondiente a dos experiencias en terreno realizadas por los alumnos bajo la coordinación de la cátedra de Epidemiología, vinculando el conocimiento teórico con la práctica en escenarios reales. Estas experiencias se incorporaron a las actividades obligatorias de la cátedra, por lo que los alumnos debían cumplir los objetivos de las mismas como parte de sus compromisos para aprobar la materia (h). Ambas experiencias buscaron relacionar la presencia de enfermedades en personas y familias que asisten a centros de primer nivel de atención de la salud, con condiciones y modos de vida que favorecen la aparición de esas enfermedades. Se propició la vivencia por parte de los estudiantes situándolos en los mismos lugares de reproducción de las familias y grupos, fuera de los muros protectores de la facultad. A pesar de tener objetivos diferentes, los dos trabajos coincidieron en la utilización de herramientas epidemiológicas para abordar problemáticas sanitarias y en la salida de los alumnos y docentes a campo.

El primero, en el año 2006, fue el Programa de Atención Nominalizada de la Salud (PANDELAS) (23): se trató de un diagnóstico sociodemográfico y sanitario de la localidad de Ensenada (Provincia de Buenos Aires), y la georreferencia de las familias con problemas sanitarios o sociales en un software informático. Los alumnos realizaron entrevistas, casa por casa, a 48.800 habitantes a fin de conocer la relación de los problemas de salud con determinantes como la estructura de las viviendas, las condiciones de vida y características del barrio en el que las personas desarrollan su vida. Luego se debatía con los propios médicos del centro de atención primaria, el rol de estos determinantes en la distribución de enfermedades. En el aula, se expusieron los problemas encontrados, así como aspectos significativos vivenciados por los estudiantes que requirieron reflexión y análisis con la guía de los docentes.

En el segundo trabajo en terreno, realizado en el año 2009, los alumnos de la misma cátedra de Epidemiología, tuvieron que realizar entrevistas a pacientes embarazadas de los centros de salud del municipio de La Plata. Muchas de estas entrevistas fueron administradas en los propios hogares de las pacientes consultantes a dichos centros. Estas pacientes fueron seleccionadas por el personal de los centros de salud, con base en la necesidad de atención evaluada por ellos. Aquí los alumnos, en grupos reducidos, debían recontactar a las pacientes con el centro de salud y, a su vez, buscar variables que relacionaran algunas características de su estado de salud con las condiciones de vida de las familias y del medio ambiente en el que vivían. En relación con los profesionales de obstetricia de los centros de salud seleccionados, trabajaron en casos en los que se combinaba el embarazo con situaciones problemáticas tales como violencia, adolescencia y pobreza, entre otras.

Se aplicaron cuestionarios semiestructurados a 411 alumnos del total de 418 pertenecientes a primer año en 2006 y a 334 de los 348 cursantes del primer año en 2009 (un total de 745 alumnos incorporados al trabajo del total de $766,97,25 \%)$. Además, para profundizar algunos aspectos relacionados a las respuestas del cuestionario, se realizaron grupos focales en 2006 y en 2009, moderados por docentes.

Mediante los cuestionarios, sumado a la técnica de grupos focales, se buscó obtener información de las ideas y representaciones de los estudiantes, antes y después de realizar el trabajo en terreno. Las dimensiones analizadas fueron:

- Nivel de prioridades en el currículo: se les pidió que ordenaran las materias por orden de importancia y que indicaran aquellos conocimientos considerados fundamentales para la práctica profesional.

- Modelo profesional: se les preguntó sobre sus intereses profesionales, cuál sería el ámbito deseado de desempeño una vez recibidos, y sus ideas acerca de aquellas especialidades médicas que deberían ser mejor remuneradas y por qué. 
- Modelos explicativos del proceso salud-enfermedad: se les preguntó por qué la gente se enferma, cómo se distribuyen los problemas de salud entre la población, cómo podemos prevenir enfermedades y cuál es el rol de los médicos frente a los problemas de salud de la población.

Una vez obtenida la información de los cuestionarios y de los grupos focales, se procedió a realizar análisis de contenido de las preguntas abiertas y de las transcripciones de los grupos focales para obtener la información correspondiente.

En forma previa se realizaron sesiones grupales para informar a los alumnos acerca de los objetivos y alcances de la investigación; se garantizó la confidencialidad y el anonimato en el manejo de los datos, siendo la participación en los cuestionarios y grupos focales absolutamente voluntaria

\section{RESULTADOS}

Las materias "duras", como se expuso en el apartado metodológico, son aquellas que tienen su base en los procesos biológicos que asientan en el cuerpo sin elementos que las relacionen con otras variables que expliquen los fenómenos de salud-enfermedad.

Un análisis estructural permite ver que del total de las 60 materias, el 70\% corresponden a la categoría "duras", relegando a las materias "sociales" al 21,6\% (el 8\% restante corresponden a los Talleres de Integración que no pueden ser incluidos en las dos categorías anteriores). Si analizamos a partir del total de horas del currículo (4.640 horas), esta desigualdad se incrementa sustancialmente Ilegando al 82\% del total de horas para las materias "duras" y un $13,3 \%$ restante para las "sociales" (el 4,7\% restante del total de horas corresponde a los Talleres de Integración) (Figura 1).

Por otro lado, al analizar la obligatoriedad como rasgo de predominancia, vemos que las materias obligatorias suman 45 , de las cuales $34(75,5 \%)$ corresponden al grupo de las materias "duras". Al calcular la cantidad de horas
(Figura 2) vemos que del total de horas obligatorias (3.830 horas), el 87,86\% corresponden a las materias "duras", quedando en un segundo plano las materias "sociales" correspondiéndoles un $6,4 \%$ del total de horas obligatorias.

Así, vemos cómo cobra mayor importancia el eje de las materias "duras", ganando terreno sobre las "sociales", tanto si medimos cantidad de materias, cantidad de horas o si las evaluamos a partir de su obligatoriedad (Figura 2).

Siguiendo con esta línea de análisis, desde el punto de vista de los recursos, tomamos como ejemplo los planteles docentes: de los 959 cargos docentes, 106 (11\%) pertenecen a las materias relacionadas a las ciencias sociales, y los 853 restantes (89\%) se reparte entre las materias "duras". En cuanto a las jerarquías docentes, el plantel de profesores (titulares y adjuntos) de las materias "sociales" constituye el $9 \%$ del total, quedando el $91 \%$ restante en las "duras" y, por último, solo el $12 \%$ de los Jefes de Trabajos Prácticos y el $18 \%$ de los Ayudantes Diplomados pertenecen a las materias con contenidos de las ciencias sociales (23), el porcentaje mayor queda en las cátedras pertenecientes a las materias "duras".

Figura 1. División porcentual de las horas del Plan de Estudios de la carrera de Medicina. Universidad Nacional de La Plata, Argentina, 2004

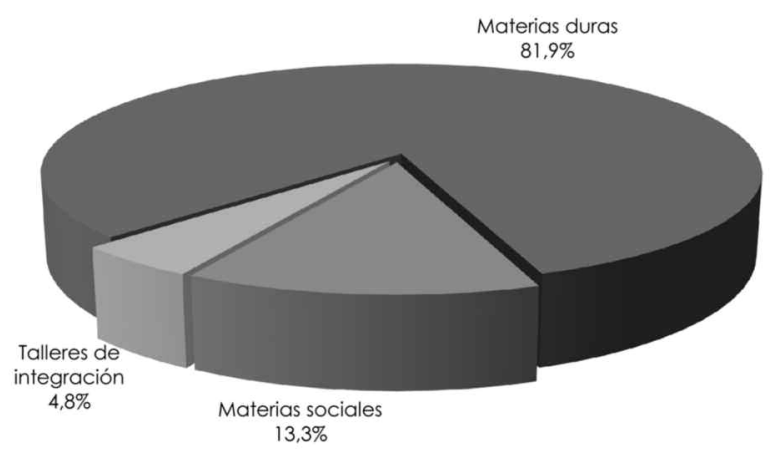

Fuente: elaboración propia a partir del Plan de Estudios de la carrera de Medicina de la Facultad de Ciencias Médicas (22). 
Figura 2. Distribución porcentual de materias, número de horas y horas obligatorias de acuerdo a su objeto de estudio. Plan de Estudios de la carrera de Medicina. Universidad Nacional de La Plata. Argentina, 2004.

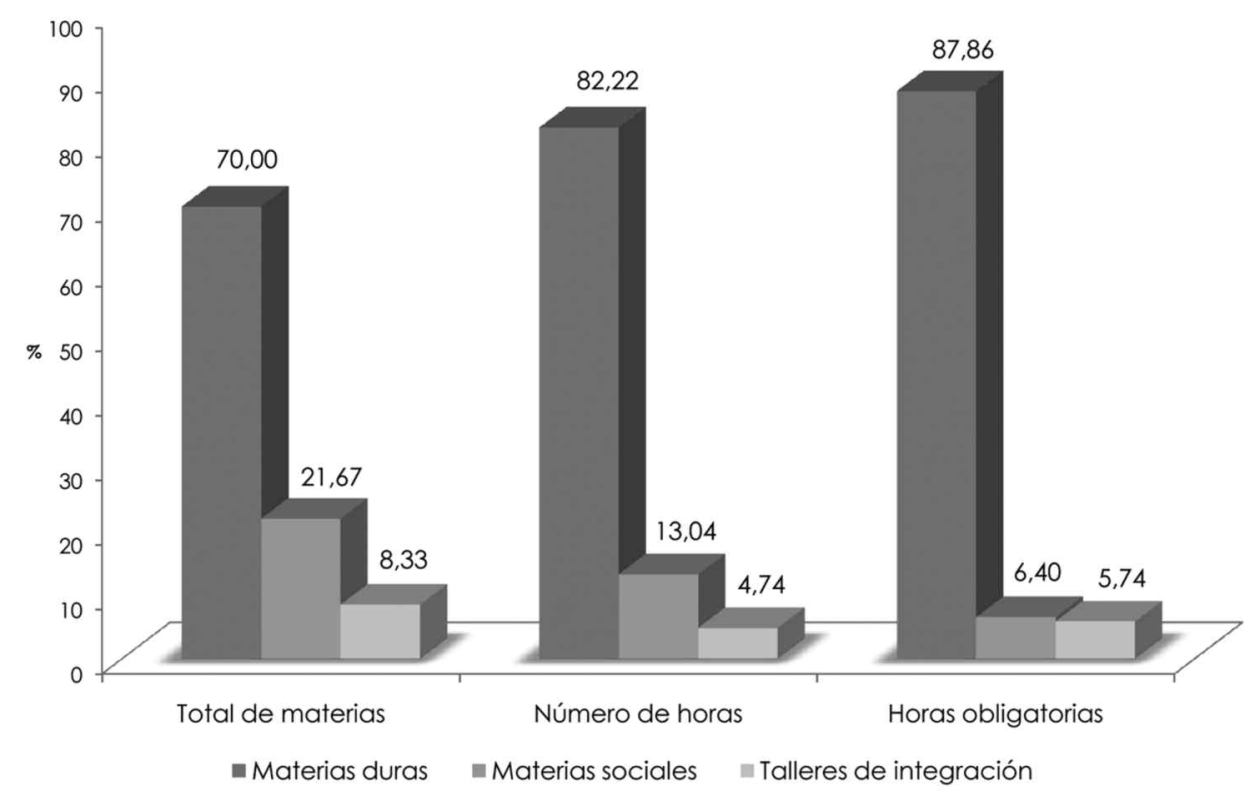

Fuente: elaboración propia a partir del Plan de Estudios de la carrera de Medicina de la Facultad de Ciencias Médicas (22).

\section{Ideas de los alumnos antes de la experiencia en terreno}

\section{Nivel de prioridades del currículo}

Esta valoración se tomó mediante la encuesta semiestructurada realizada a los alumnos el primer día de clases, en la que debían realizar un listado de materias por orden de importancia. En la misma se observa el prestigio que tienen las materias de la rama biológica por sobre las sociales, ya que las materias vinculadas con la rama social aparecen ubicadas en promedio en el puesto 26 . De los 745 encuestados, solamente el $5 \%$ de los alumnos ubicó una materia relacionada a lo social dentro de las 10 primeras en prioridad. Un 15\% ubicó en el último lugar a una de estas materias.

Sumado a esto, en la dinámica de desarroIlo de sus estudios, estas asignaturas resultan ser consideradas más importantes para los alumnos, ya que son las necesarias para mantener la regularidad y para poder pasar de año. El resultado de estas situaciones genera en el ideario de los alumnos la existencia de materias de "primer y segundo orden de importancia".

Cuando se indagó acerca de estos resultados en los grupos focales aparecieron una serie de cuestionamientos acerca de la forma en que se dan estas materias y surgieron frases como "son aburridas", "no sirven para curar a las personas", "no se ponen de acuerdo entre las cátedras" (haciendo alusión a que diferentes autores pueden tomar en forma diferente los conceptos del campo social, o al menos no estrictamente iguales, a diferencia de las materias biológicas). También relacionaron estas materias con cierta facilidad, ya que consideraban que articulando algunos conceptos acerca de factores relacionados a problemas sociales se da por sabida la materia.

\section{Modelo profesional}

En el cuestionario semiestructurado realizado a los alumnos en el ingreso a la facultad, 
se les realizaron preguntas acerca del imaginario del desarrollo profesional, en la que todos sin excepción mencionaron áreas de desempeño asistencial exclusivo, ligado en su mayoría a especialidades y subespecialidades. De los 745 alumnos encuestados en 2006 y 2009, el 35\% (261 alumnos) eligió especialidades (cirugía, pediatría, clínica, oftalmología, etc.), mientras que el $65 \%$ restante eligió subespecialidades (terapia neonatal, cirugía plástica, neurocirugía, endocrinología infantil, etc.). Lo interesante de estos resultados es que los que eligieron especialidades refirieron que lo hacían con un espíritu de "ayuda", de servicio a la gente, mientras que los que optaron por subespecialidades lo vincularon más al prestigio o al rédito económico. También esta división de servicio/prestigio, se observa con relación al género, mientras que las mujeres optaron más por las especialidades $(68 \%$ de las mujeres) y vincularon el trabajo con el servicio, los hombres en mayor medida se volcaron a las subespecialidades $(75 \%)$. Es interesante observar que tanto los que eligieron especialidades como los que se inclinaron por las subespecialidades, imaginaron como lugar de trabajo al sector privado u hospitales públicos en el mejor de los casos. En ningún momento surgió la idea de los centros de salud como posible lugar de desempeño laboral. Cuando se indagó acerca de cuál debe ser la remuneración entre las diferentes especialidades, ordenándolas de mayor a menor, la rama de las cirugías fue la que se llevó los mayores dividendos, seguida por las subespecialidades y por último, las demás especialidades. Siempre surgió la idea del quirófano en donde la vida del paciente está "en las manos del médico" y "no se pueden cometer errores", asociando estas ideas con una mayor remuneración.

\section{Modelos explicativos del proceso salud-enfermedad-atención}

En este punto se buscó recoger las ideas que tenían los alumnos sobre los modelos explicativos de las enfermedades, antes de la salida a terreno. En las comisiones se intentó generar el debate mediante grupos focales proponiendo que se discutiera acerca de las "causas" que favorecen la aparición de enfermedades. Es interesante ver cómo la discusión se desarrolló básicamente en torno de las causas estrictamente biológicas: del cuerpo como agente dañado, órganos que se deterioran, gérmenes que invaden el cuerpo, personas siempre recostadas en una cama de hospital, en general graves, y en las que su vida depende exclusivamente del correcto accionar del médico y sus habilidades. Solo en casos extremos, como la desnutrición infantil, aparecieron las condiciones económicas, sociales y educativas en la explicación. Pero allí surgió la idea de que la medicina tiene poco o nada que hacer, no existen tratamientos o medicamentos que solucionen lo social, por lo tanto no pertenece a su ámbito de acción, siempre enmarcado en el terreno de las generalidades y de la imposibilidad de accionar en otro sentido que no sea el estrictamente asistencial, "...nosotros vamos a poder diagnosticar y tratar, en lo social ya no podemos hacer nada" (Alumna, 19 años).

\section{Ideas de los alumnos después de la experiencia en terreno}

\section{Nivel de prioridades del currículo}

En términos generales todos los grupos coincidieron en que la salida a terreno fue positiva porque les permitió conocer realidades sociales desconocidas personalmente, además de incorporar fuertemente la idea de los centros de salud como lugares de práctica profesional. Por supuesto que siguió latente la tensión con las materias "duras", ya que en las salidas a terreno aparecieron comentarios como: "...se pierde mucho tiempo [...] los lugares quedan lejos [...] con ir un par de veces estaría bien" (Alumno, 20 años), haciendo referencia al lugar secundario de este tipo de experiencias con relación a lo que para los alumnos es importante (anatomía, fisiología, bioquímica, etc.).

En cuanto al nivel de prioridades del currículo, luego de la experiencia, los alumnos siguieron ubicando a las materias sociales en puestos de baja importancia (puesto 25). En este punto los cambios se observaron en los grupos focales mediante expresiones como "importancia de los problemas sociales [...] concientización de los médicos [...] los compañeros que no vieron esto, sería importante que lo hagan" (Alumna, 19 años). 
Si bien estas expresiones se enmarcan dentro de una suerte de vocación benéfica y no dentro de las responsabilidades profesionales, se nota que la experiencia los ha hecho reflexionar y criticar los límites de la formación clásica.

\section{Modelo profesional}

En torno al modelo profesional, no han modificado su idea de la especialidad a seguir, salvo en un pequeño número de casos, lo que sí se ha modificado es que cobra existencia y relevancia el profesional que trabaja en los centros de salud de primer nivel, y se estima que su paga debiera ser igualada a las demás especialidades. Esto, si bien genera una apertura en torno a la igualdad en la remuneración, no tiene impacto en el prestigio, ya que muy pocos alumnos son los que incorporan al primer nivel como su lugar de desarrollo profesional.

\section{Modelos explicativos del proceso salud-enfermedad-atención}

Luego de la experiencia, el mayor impacto se observó en la función de los modelos explicativos del PSEA. Aquí los alumnos se plantearon el peso real de los problemas sociales sobre los fenómenos de salud y enfermedad. También se observó como positivo el cambio de percepción en torno a la responsabilidad sobre estos problemas de salud, sacándolos del ámbito personal/individual hacia otras instituciones sociales con poder (gobierno, Estado, políticos, sistema económico). Acompañando a esta idea, se observó la necesidad manifiesta de tener herramientas de las ciencias sociales para dar cuenta de dichas problemáticas complejas. Este es otro cambio importante que da al profesional médico una responsabilidad real en la problemática, al incorporar el problema al área de su incumbencia y considerar los aportes de las ciencias sociales como herramientas válidas para el posterior desarrollo profesional.

Se comienzaron a dar explicaciones rudimentarias acerca de conceptos sociales "...las mujeres pobres, sin educación tienen hijos cada vez más jóvenes, así no pueden estudiar, y no les queda otra que trabajar de lo que sea, tienen más hijos y ya los crían pobres" (Alumna, 19 años).

\section{DISCUSIÓN}

Es posible observar, en los últimos treinta años, una tendencia mundial estable hacia la formación de profesionales especializados y superespecializados en disciplinas médicas. Según datos del Ministerio de Salud de la Provincia de Buenos Aires (24), las residencias en las que quedan cargos sin ocupar son aquellas denominadas básicas: pediatría, clínica y medicina general. Mientras tanto, las vacantes son insuficientes en aquellas residencias relacionadas con una mayor complejidad: neonatología, terapia intensiva, anestesiología, entre otras. Esos aspectos pueden ser tomados como la vigencia y plenitud del $\mathrm{MMH}$ en el campo de la salud, ya que la tendencia a la superespecialización es parte sustancial de las características de dicho modelo.

Para modificar y contrarrestar esa hegemonía, se hace necesario reorientar el perfil profesional del egresado de las escuelas de medicina incorporando visiones que contemplen los determinantes sociales y las realidades colectivas. Esta reorientación no debe basarse exclusivamente en cambios de contenidos curriculares, sino que deben acompañarse de estrategias pedagógicas que impacten en las diferentes dimensiones de la formación -en lo actitudinal, en las prácticas y conocimientos- y que tengan un importante aporte de las ciencias sociales que, frente a una concepción puramente biologicista, posibilitan generar un conflicto cognitivo (25) mostrando la superficialidad de los esquemas tradicionales y sus déficits explicativos. Las ciencias sociales permiten incorporar la complejidad que complementa y enriquece estos esquemas. Por ello, la visión de la medicina y de la salud se vuelve más compleja: no se trata de anexar materias de las ciencias sociales a la línea "dura", sino de revisar la concepción general que se tiene de la salud en la totalidad del plan de estudios.

El nuevo perfil del egresado de la Facultad de Ciencias Médicas de la UNLP establece formalmente la necesidad de formar un profesional comprometido con la realidad socioeconómica del país, que incorpore conocimientos, prácticas y actitudes que permitan darle al fenómeno de salud y enfermedad la complejidad que presenta (26). 
Sin embargo, no hay ningún dato de la realidad académica que permita avizorar la estrategia educativa para lograr ese "producto" final. La realidad es un tanto diferente, ya que el tipo de inserción laboral predominante en los nuevos profesionales se dirige a la superespecialización de la práctica, lo cual es reforzado por los modelos de rol del staff docente.

Se observa una contradicción -con múltiples interpretaciones- entre lo que pretende formar el currículo, expresado en el perfil profesional, y lo que finalmente resulta como producto final: el egresado. Con relación a ello, vemos que los conocimientos y materias que se vinculan con disciplinas de las ciencias sociales (sociología, antropología e historia) que forman parte de los capitales que portan los pretendientes del campo, son consideradas como accesorias $-\mathrm{y}$ hasta marginales- por los actores dominantes en la formación del profesional médico.

Además, se observa en los alumnos, ante ciertos problemas de salud como la obesidad, los problemas cardiovasculares y los niños sin vacunación, una tendencia a culpabilizar al paciente; tendencia que reproduce el modelo hegemónico individualista y ahistórico, impidiendo la cabal comprensión del problema de salud. Esta actitud sería factible de ser modificada mediante la enseñanza y puesta en práctica de herramientas de las ciencias sociales. Alcanzar un perfil de profesional de la salud con sentido social, requeriría un cambio en la relación de fuerzas de los actores del campo, ya que implicaría introducir espacios de crítica a los modelos profesionales hegemónicos. Por supuesto que esto supone confrontaciones y lucha en torno de los distintos capitales en juego al interior del campo de la salud.

La experiencia de prácticas en terreno que hemos descrito muestra el impacto positivo a nivel de modificar modelos explicativos de los PSEA que tenían los alumnos, generando aperturas hacia explicaciones más complejas luego de las experiencias vividas y la discusión generada por dicha experiencia.

La posibilidad de que los alumnos incorporen la complejidad de los PSEA, así como la necesidad de analizarlos en función de un contexto que lo determina, es un punto crucial a la hora de avanzar en la discusión del modelo médico para modificarlo.
En nuestra experiencia, no se han observado cambios sustanciales en los niveles de prioridades en el currículo médico definidos por los alumnos luego de haber atravesado la práctica, a pesar de que refirieron necesitar conocimientos de las ciencias sociales para dar sentido al origen de los PSEA. Esta situación tampoco se vio reflejada en modificaciones en la relevancia de estas materias y su distribución a lo largo de la carrera. Resulta de una gran complejidad la relación entre las expectativas de formación de los alumnos, la realidad que ofrece la estructura curricular de la carrera y las necesidades del perfil profesional actual. Si bien los alumnos ya ingresan a la formación con expectativas coincidentes a lo que ofrece el currículo, se deben potenciar espacios de crítica en ellos que reorienten sus expectativas a las necesidades sanitarias. Este debate debería formar parte de una discusión más amplia en la que todos los actores estén representados; una discusión acerca de los fines de la enseñanza y su vinculación con la práctica profesional en el contexto social y político en los que se desarrollan.

En cuanto a la valoración de las especialidades y superespecialidades por parte de los alumnos, la práctica desarrollada no modificó el orden de importancia que asignan a las superespecialidades en primer lugar, seguidas de cerca por las especialidades hospitalarias, muy por encima de aquellas especialidades asociadas al primer nivel. Este rasgo, característico del modelo médico actual, debería ser modificado, para lo cual proponemos trabajar en la articulación entre materias y conocimientos del ciclo clínico y las materias relacionadas con aspectos sociales, de manera tal que se equiparen en importancia, prestigio y estructura.

El dato que sugiere un cambio en la categoría prestigio profesional es la modificación de la remuneración -quedando de manifiesto la relación entre capital económico y capital simbólico-, por lo que sería importante igualar el salario de los profesionales del primer nivel de atención con los de los demás profesionales. También apareció en la idea de los alumnos el primer nivel de atención como escenario posible de la práctica profesional que a priori se encontraba ausente. Es sumamente importante este aspecto en cuanto a la posibilidad de contar con profesionales que respondan a las necesidades de las políticas públicas. 
En las dos experiencias descritas, realizadas en diferentes años y con diferentes alumnos, no hemos hallado alguna de las barreras a la implementación de actividades de campo descriptas por otros autores (27). A pesar de realizar las prácticas en locaciones alejadas del casco urbano, e incluso en otras localidades, no fue un punto importante de discordia entre los alumnos. Tal como remarcamos, surgió solo en el momento en que debían dedicarse a otras materias. Tampoco fue un tema de debate la seguridad de los mismos en relación con las zonas marginales (el contacto y apoyo de autoridades locales y policiales hizo que este temor no apareciera).

\section{CONCLUSIONES}

El campo de la salud tiene varios escenarios de disputa; uno de ellos es, claramente, la formación de profesionales en las escuelas de medicina. Estos profesionales -formados y en formación- tienen incorporado el modelo de profesional hegemónico, ya que es en la formación donde se da esta incorporación (i), que no es más que la producción y reproducción del habitus médico liberal, acrítico, que basa su práctica en un modelo biologicista. Este es el modelo vigente y mantenerlo o transformarlo es el objeto de la disputa.

Siguiendo con esta lógica, la postura de los actores que intentan promover otro modelo de profesional, debe apuntar a la enseñanza en tanto instancia que posibilita la modificación de ese habitus, ya que es allí, en la formación médica de grado, donde el mismo se estructura (28). Para los actores tradicionales, sostener el modelo actual es una manera de sostener esa naturalización que, por un lado, les permite tener el apoyo tácito e incorporado de la mayoría de los miembros del campo y, por otro, como ya mencionamos, mantener sus propias posiciones de poder.

En este sentido, se observan numerosas instituciones, tanto en Argentina, como la Universidad Nacional de La Plata (UNLP), la Universidad Nacional del Sur (UNS), la Universidad Nacional de Lanús (UNLA), la Universidad Nacional de Rosario (UNR), como en el exterior, por ejemplo, la Universidad Nacional Autónoma de México (UNAM), u otras instituciones como la propia Organización Panamericana de la Salud, que se encuentran trabajando en la elaboración de estrategias educativas para reorientar el perfil profesional (29). Sin embargo, estos intentos han reportado dificultades en el cumplimiento de sus metas, ya que las instituciones y corporaciones profesionales, a través de mecanismos de reproducción e inculcación (congresos médicos, "premios" de los laboratorios farmacéuticos) siguen sosteniendo egresados funcionales a sus intereses (fragmentarios, positivistas, individualistas y biologicistas).

A lo largo del artículo describimos lo que consideramos una experiencia significativa en el proceso de formación de profesionales, que debe ser comprendida en el marco de esa disputa. Este tipo de experiencias estimulan reflexiones propias de los alumnos y no "naturalmente" impuestas por los actores dominantes del campo.

Un punto muy importante a analizar en futuras investigaciones es el papel y el peso que tienen las características de los docentes (trayectoria laboral, profesión, tipo y grado de formación), debido a que se observó por parte de algunos docentes -cuyos sitios habituales de trabajo son diametralmente opuestos a lo que se vivió en terreno- algunos temores con relación a la seguridad, aspectos que afortunadamente no limitaron la experiencia (30). Por ello, sería importante evaluar los intereses de los docentes, e incorporar a la enseñanza a profesionales que ya estén trabajando en esos lugares. Esto permitiría disminuir las resistencias de los propios docentes -resistencias ante la incertidumbre de ingresar a un ámbito desconocido, pero también resistencias ante los cambios en su mundo internalizado de certezas-, aceitando los mecanismos de entrada y permanencia en el escaso tiempo disponible para la práctica y modificando en los mismos docentes las actitudes naturalizadas que impiden modificaciones en el perfil profesional.

La propuesta de enseñanza que analizamos debe entenderse como parte de la acumulación de capital simbólico y social de los actores que, teniendo como objetivo primario la formación en las escuelas de medicina, aspiran a modificar las relaciones de fuerza en el campo de la 
salud. Para lograr este objetivo, es necesario transformar el modelo de profesional médico, tarea que no es simple porque pone de relieve los distintos intereses que se disputan en el campo. Sin embargo, a pesar de que la tarea no es simple, es necesaria.

\section{AGRADECIMIENTOS}

A la Dra. Glenda Morandi, de la carrera de Especialización Docente de la Universidad Nacional de La Plata por su apoyo y guía permanente. A los compañeros de cátedra que siguieron la propuesta de manera entusiasta.

\section{NOTAS FINALES}

a. Campo: En términos analíticos, un campo puede definirse como una red o una configuración de relaciones objetivas entre posiciones. Estas posiciones se definen objetivamente en su existencia y en las determinaciones que imponen a sus ocupantes, agentes o instituciones, por su situación (situs) actual y potencial en la estructura de distribución de los diferentes tipos de poder (o de capital) cuya posesión gobierna el acceso a los beneficios específicos que están en juego en el campo y, al mismo tiempo, por sus relaciones objetivas con las otras posiciones (dominación, subordinación, homología, etc.)

b. En la lógica de Bourdieu es posible distinguir cuatro tipos de capital: 1) El capital económico, constituido por los diferentes factores de producción (tierras, fábricas, trabajo) y el conjunto de los bienes económicos: ingreso, patrimonio, bienes materiales. 2) El capital cultural, correspondiente al conjunto de las calificaciones intelectuales, sean producidas por el sistema escolar o transmitidas por la familia. 3) El capital social se define en esencia como el conjunto de las relaciones sociales de las que dispone un individuo o grupo. 4) El capital simbólico corresponde al conjunto de los rituales (como la etiqueta o el protocolo) ligados al honor y el reconocimiento.

c. "Los campos se presentan para la aprehensión sincrónica como espacios estructurados de posiciones (o de puestos) cuyas propiedades dependen de su posición en dichos espacios..." Así comienza Bourdieu su texto "Algunas propiedades de los campos" (5). Esos puestos son los de pretendientes y dominantes y en cada campo específico las luchas entre ellos toman formas diferentes. d. Es interesante ver cómo los actores del campo se apropian de las teorías elaboradas sobre el campo mismo, en lo que se denomina "efecto de teoría", para un análisis de cómo el modelo de Menéndez se convierte en teoría nativa de los actores del campo de la salud (7).

e. Con espiral reproductivista hacemos referencia a la idea de una relación que mutuamente se potencia generando un crescendo de sus efectos.

f. Estas dos posiciones tan dicotómicas -la minoría y los herederos de la tradición- se distinguen solo a fines analíticos, para que quede clara la disputa dentro del campo. Esto no significa desconocer que, en el campo de la salud, se distinguen posiciones intermedias que agrupan a un abanico amplio de profesionales, asociaciones, etc.

g. Las categorías "duras" y "sociales" son las que utilizan los actores del campo en el cotidiano para clasificar las materias.

h. La participación en las prácticas en terreno por parte de los alumnos fueron obligatorias en el marco de las actividades para aprobar la cursada. Su participación como informantes de la investigación, ya sea completando los cuestionarios o participando de grupos focales, fue voluntaria.

i. Aludir a incorporación hace referencia a la adquisición que se da durante la formación de habitus profesional, lo que no quita que la formación médica esté acorde con las representaciones sociales acerca de los médicos que traen consigo los estudiantes al ingresar a la carrera. 


\section{REFERENCIAS BIBLIOGRÁFICAS}

1. Hasenfeld Y. Enfoques teóricos sobre las organizaciones de servicios humanos. En: Organizaciones al servicio del hombre. México DF: Fondo de Cultura Económica; 1990. p. 22-62.

2. Cordera-Campos R, Sheinbaum-Lerner D. Los retos de la autonomía universitaria en la sociedad del conocimiento. Universidades [Internet]. 2008 [citado 28 abr 2011];58(36). Disponible en: http://redalyc.uaemex.mx/redalyc/src/inicio/ArtP dfRed.jsp?iCve $=37312909010$.

3. Spinelli H. Las dimensiones del campo de la salud en Argentina. Salud Colectiva. 2010;6(3):275293.

4. Bourdieu P, Wacquant L. Una invitación a la sociología reflexiva. Buenos Aires: Siglo XXI Editores; 2005.

5. Bourdieu P. Algunas propiedades de los campos. En: Sociología y Cultura. México DF: Conaculta y Grijalbo: 1990. p. 109-114.

6. Menéndez EL. El modelo médico y la salud de los trabajadores. Salud Colectiva [Internet]. 2005 [citado 16 sep 2011];1(1):9-32. Disponible en: http://www.scielo.org.ar/scielo.php?script = sci_arttext\&pid = S1851-82652005000100002\&lng = es.

7. Pozzio M. De soldados a espías: resignificaciones y conflictos en torno de la definición de APS en la implementación de políticas públicas. En: Bohoslavsky E, Soprano G, editores. Un Estado con rostro humano: Funcionarios e instituciones estatales en la Argentina (1880 a la actualidad). Buenos Aires: Prometeo, Universidad Nacional de General Sarmiento; 2010.

8. Linares-Pérez N, López-Arellano O. La equidad en salud: propuestas conceptuales, aspectos críticos y perspectivas desde el campo de la salud colectiva. Medicina Social. 2008;3(3):247-259.

9. Spinelli H. Laberintos. Salud Colectiva [Internet]. 2005 [citado 13 abr 2012]; 1(1):7-7. Disponible en: http://www.scielo.org.ar/scielo. php? script =sci_arttext\&pid=S1851$82652005000100001 \& \operatorname{lng}=$ es.

10. Minayo MCS. El desafío del conocimiento: Investigación cualitativa en salud. Buenos Aires: Lugar Editorial; 1997.

11. Bourdieu P, Passeron JC. La reproducción: Elementos para una teoría del sistema de enseñanza. México DF: Distribuciones Fontamara; 1996.
12. Menéndez EL. Morir de alcohol. En: Saber y hegemonía médica. México DF: Conaculta, Alianza; 1990.

13. Bourdieu P. El sentido práctico. Madrid: Taurus; 1993.

14. García JC. La educación médica en América Latina. Washington: OPS, OMS; 1972. (Publicación Científica No 255).

15. Brissón ME, Galli A. Conferencia Argentina de Educación Médica: agendas, aportes y temas emergentes. Educación Médica [Internet]. 2005 [citado 15 abr 2012];8(1):38-47. Disponible en: http://scielo.isciii.es/pdf/edu/v8n1/original4.pdf.

16. Merhy EE, Feuerwerker L, Ceccim R. Educación permanente en salud: una estrategia para intervenir en la micropolítica del trabajo en salud. Salud Colectiva. 2006;2(2):147-160.

17. Castro R. Habitus profesional y ciudadanía: hacia un estudio sociológico sobre los conflictos entre el campo médico y los derechos en salud reproductiva en México. En: Castro $R$, López Gómez A, editores. Poder médico y ciudadanía: el conflicto social de los profesionales de la salud con los derechos reproductivos en América Latina. Avances y desafíos en la investigación regional. México DF: UNAM, CRIM; 2010. p. 49-72.

18. Lahire B, compilador. El trabajo sociológico de Pierre Bourdieu. Deudas y críticas. Buenos Aires: Siglo XXI Editores; 2005.

19. Organización Panamericana de la Salud. Metas regionales en materia de recursos humanos para la Salud 2007-2015 [Internet]. 27a Conferencia Sanitaria Panamericana; 59a sesión del Comité Regional. Washington DC: OPS, OMS; 2007 [citado 12 sep 2011]. Disponible en: http://devserver.paho.org/observatoriorh/drupal/s ites/default/files/webfiles/fulltext/OPS 27 CSP Metas_Recursos_Humanos_2007.pdf.

20. Scott JW. Experiencia. La Ventana. 2001; (13):42-73.

21. Candreva A, Morandi G. El currículum universitario: Entre la teoría y la práctica. En: García Santa María MT, coordinadora. Un currículum de ciencias sociales para el siglo XXI: qué contenidos y para qué. Sevilla: Diada Editora; 1999.

22. Universidad Nacional de La Plata, Facultad de Ciencias Médicas. Plan 2004: Listado de asignaturas de la carrera [Internet] 2004 [citado 15 abril 2012]. Disponible en: http://www.med. 
unlp.edu.ar//plan estudio.php?itemc $=$ Plan $\% 202$ $004 \&$ idc $=7 \&$ idl $=25 \&$ idbot $=3$.

23. Marin GH, Silberman M. Programa de Atención Personalizada y Nominal de la SaludPANDELAS Buenos Aires, Argentina, 2006. Revista de Salud Pública [Internet]. 2008 [citado 10 abr 2012];10(2):203-214. Disponible en: http://www.scielosp.org/scielo.php?pid = S0124$00642008000200001 \&$ script $=$ sci arttext.

24. Dirección de Capacitación de Profesionales de la Salud, Ministerio de Salud de la provincia de Buenos Aires. Anexo: Cupos de residentes ciclo 2012 [Internet]. 2012 [citado 15 abr 2012]. Disponible en: http://www.ms.gba.gov.ar/ssps/ Residencias/Cupos_2012.pdf.

25. Sánchez-Inesta T. La construcción del aprendizaje en el aula. Buenos Aires: Magisterio del Río de la Plata; 1995. (Colección Respuestas Educativas).

26. Facultad de Ciencias Médicas, UNLP. Perfil del médico [Internet]. 2012 [citado 15 abr 2012].
Disponible en: http://www.med.unlp.edu.ar/ secretaria_asuntos_academicos.php? idbot $=2 \&$ it emi $=$ perfil_medico.

27. Cronholm PF, Margo K, Bazelon G, Bream K, Benett IM, Barg FK. Student attitudes: potential barriers to implementing a community medicine field activity. Family Medicine. 2009;41(1):22-27.

28. Bourdieu P. Homo academicus. Buenos Aires: Siglo XXI Editores; 2008.

29. Organización Panamericana de la Salud. La Formación en Medicina orientada hacia la Atención Primaria de Salud. Washington: OPS; 2008. (Serie Renovación de la Atención Primaria de Salud en las Américas No 2).

30. Davis WK, Jolly BC, Page GC, Rothman AI, editors. Moving medical education from the hospital to the community: Report of the Seventh Cambridge Conference on Medical Education. Ann Arbor: University of Michigan Medical School; 1995.

\section{FORMA DE CITAR}

Silberman MS, Silberman P, Pozzio M. Evaluación de una propuesta pedagógica de enseñanza de la Medicina. Salud Colectiva. 2012;8(2):175-189.

Recibido el 6 de junio de 2011

Versión final presentada el 18 de septiembre de 2011

Aprobado el 28 de diciembre de 2011 